\title{
El impacto del Informe PISA en la sociedad española: el caso de la prensa escrita
}

The impact of PISA in the Spanish society: the case of written press

\author{
González-Mayorga, Héctor; Vidal, Javier \& Vieira, María José \\ Universidad de León (España)
}

\begin{abstract}
Resumen
En la última década los resultados de los diferentes Informes PISA han avivado el debate sobre la calidad de nuestro sistema educativo, repercutiendo en la opinión pública y en la toma de decisiones en el plano político. En el presente estudio se analiza el impacto que han tenido los resultados de PISA en la sociedad española a través de las noticias publicadas en el periódico «El País» desde el 2001 al 2014, atendiendo a las siguientes cuestiones: a) los subgéneros periodísticos utilizados para hablar de PISA y su autoría; b) la evolución de las noticias desde PISA 2001 hasta PISA 2012; y c) la frecuencia de uso de las competencias evaluadas por PISA como argumento informativo. Los resultados muestran la gran trascendencia de la evaluación PISA en prensa, sobre todo desde la edición del Informe correspondiente al año 2003 y reflejado en su mayoría en artículos de carácter informativodescriptivo. Por otro lado, se evidencia el tratamiento que hacen los medios de comunicación escrita a las competencias matemática, científica y lectora, dando una mayor relevancia a esta última en los últimos años. Por último, se concluye que la prensa escrita ha sido un gran contribuyente a la hora de trasladar los resultados obtenidos en PISA a la opinión pública, a pesar del uso inadecuado que se hace en ocasiones de los datos sin tener en cuenta las limitaciones del propio estudio.
\end{abstract}

Palabras clave: Impacto, PISA, competencias, prensa, educación y medios, debate, opinión pública, uso de la información.

\begin{abstract}
In the last decade, the results of the Programme for International Student Assessment (PISA) have increased the debate about the quality of the Spanish educational system, reverberating in the public opinion and the policy decision making. This study aims to analyse the impact the results of PISA in the Spanish society through the articles which have appeared in «El País» (the non-sport newspaper with the biggest circulation in Spain) from 2001 to 2014, attending to the following issues: a) the press subgenres used to talk about PISA and its authorship; b) the evolution of news from PISA 2001 to PISA 2012; and c) the frequency of use of the skills assessed by PISA as an information argument. The results of our study show the great importance of PISA results in press, especially since the 2003 edition, which was mostly reflected in descriptive and informative texts. On the other hand, the use that media makes of the competences in mathematics, science and reading is evidenced, giving a greater relevance to this last one in recent years. Finally, we conclude that the press has been a great contributor when it comes to the transfer of PISA results to the public opinion, in spite the inappropriate use that is sometimes made of data without considering the limitations of the study.
\end{abstract}

Fecha de recepción 2016 October 10

Fecha de aprobación 2017 February 8

Fecha de publicación 2017 February 9

Reception Date 2017 Octubre 10

Approval Date 2017 Febrero 8

Publication Date: 2017 Febrero 9

Keywords: Impact, PISA, competences, press, education and media, debate, public opinion, use of data.

En los últimos años se ha podido observar un creciente interés en analizar la calidad de los diferentes sistemas educativos, a juzgar por la implementación de programas de evaluación de los mismos como el Estudio de las Tendencias en Matemáticas y Ciencias (TIMSS), el Estudio Internacional de Progreso 
en Comprensión Lectora (PIRLS) o el Programa Internacional para la Evaluación de Estudiantes (PISA). Las características que distinguen a PISA de otras evaluaciones son: (a) el carácter orientador hacia la toma de decisiones en política educativa; (b) el compromiso de aplicación regular cada tres años (TIMSS es cuatrienal y PIRLS quinquenal); (c) su naturaleza participativa y colaborativa, puesto que en él colaboran instituciones internacionales proporcionando un soporte técnico, científico y pedagógico; y, por último, (d) su amplia cobertura geográfica y su rigurosidad metodológica (Calero \& Choi, 2012; OCDE, 2002).

No obstante, algunos autores han señalado algunas limitaciones entre las que podemos destacar: (a) el diseño transversal del estudio, que no permite medir la causalidad; (b) la falta de adecuación de los ítems del estudio en términos transculturales; (c) el hecho de que se evalúe en una muestra de sujetos siguiendo un criterio de edad y no de curso; y (d) la falta de control de variables extrañas como puede ser la motivación del alumnado a la hora de realizar la evaluación o que éstos puedan recibir un entrenamiento específico para llevar a cabo la prueba (Bautier \& Rayou, 2007; Pedró, 2012). Otros ponen de manifiesto la opacidad que caracteriza a este tipo de evaluaciones, a pesar de los esfuerzos de los responsables de PISA por poner a disposición de la sociedad manuales, guías y bases de datos (Domínguez, Vieira \& Vidal, 2012; Pérez y Soto, 2011; Rutkowski \& Rutkowski, 2010).

Los resultados de PISA tienen consecuencias en políticas socio-educativas. Schleicher (2006, 2016) describe que el principal objetivo de PISA es permitir a los responsables políticos observar qué factores están asociados al éxito educativo, animando a estos a no establecer simples comparaciones entre los resultados, sino a ir más allá. Para ello, los resultados se presentan en cuatro grandes grupos temáticos: la calidad de los resultados, igualdad y equidad entre resultados $\mathrm{y}$ oportunidades educativas, la eficacia $\mathrm{y}$ eficiencia de los procesos educativos, y el impacto de los resultados en el bienestar social y económico (Ministerio de Educación y Cultura, 2001; Ministerio de Educación y Ciencia, 2007; Ministerio de Educación, Política Social y Deporte, 2008; Ministerio de Educación, 2010; Ministerio de Educación, Cultura y Deporte, 2012). Asimismo, Calero y Choi (2012) señalan que la importancia de PISA reside en torno a las finalidades para las que ha sido creada, siendo éstas: la elección eficaz por parte de las familias, la rendición de cuentas por parte de los centros públicos, la proporción de información interna para los centros docentes, el suministro de información a la investigación educativa y la obtención de información general del funcionamiento del sistema educativo. En este sentido, países como Alemania, Suecia o Reino Unido son buenos ejemplos de cómo los gobiernos han utilizado los resultados para reformular sus políticas y aplicar amplias reformas en sus sistemas educativos (Pons, 2012; Robert, 2010; Simola, 2013).

Un indicador del impacto de las evaluaciones externas es su presencia en los medios de comunicación. Stack (2007) analizó la cobertura que hicieron diversos medios a los resultados de TIMSS en 1999, y PISA en 2000 y 2003; mientras que otros como Liegmann y van Ackeren (2012) analizaron el impacto de los resultados de PIRLS 2006 en doce países, incluyendo su tratamiento en la prensa escrita. Pons (2012) describió cómo el impacto en los medios de los resultados de PISA puede utilizarse como un arma de debate político, dejando de lado la verdadera relevancia educativa que una evaluación de tal magnitud pueda tener. En España encontramos algunos estudios sobre el impacto de PISA en la prensa en Ferrer y Massot (2005), y Massot, Ferrer y Ferrer (2006), quienes concluyen que la prensa hace un uso político inadecuado, no aportando información detallada y precisa sobre qué supone PISA desde un punto de vista metodológico, lo que contribuye a que los lectores formen una imagen negativa de las evaluaciones educativas externas, en general, $\mathrm{y}$ de PISA en particular. 
Es, por tanto, incuestionable que los medios de comunicación escrita son uno de los principales medios de difusión de los resultados e implicaciones que tiene PISA tanto a nivel académico como político y social. Por ello, el presente estudio se centra en describir y analizar el impacto de los resultados de PISA en la sociedad a través de las noticias aparecidas en la prensa escrita. Dicha descripción y análisis se han concretado en los siguientes apartados: a) descripción de cuáles han sido los principales subgéneros periodísticos utilizados para hablar de PISA y quién ha escrito dichas noticias; b) descripción de la evolución de las noticias; y c) descripción de cuáles han sido las competencias evaluadas por PISA más utilizadas como argumento a la hora de redactar las noticias.

\section{Método}

\section{Muestra}

La muestra se compone de 778 noticias, extraídas de la edición digital del diario «El País» y correspondientes al intervalo existente entre el 31 de diciembre de 2001 y 2 de diciembre de 2013, ambas inclusive. La elección de «El País» como unidad de análisis se justifica por el hecho de ser el periódico no deportivo de tirada nacional con mayor número de lectores diarios, según los diversos resúmenes generales de resultados del Estudio General de Medios (EGM) ${ }^{1}$ correspondientes al periodo comprendido entre abril de 2000 y marzo de 2014. Por otro lado, se ha realizado el análisis con un único medio de difusión para centrarse en la evolución y eliminar la influencia de otras variables como línea editorial o características del medio.

\section{Recogida de datos}

La recogida de datos se realizó a través del buscador de contenido de la edición digital del periódico «El País» (http://goo.gl/V0ljt3) utilizando las palabras clave «Informe PISA» e «Informes PISA». Además, incluyeron aquellos otros artículos etiquetados por el propio periódico bajo la categoría de «Informes PISA» (http://goo.gl/YQNgvd). Se seleccionaron aquellos artículos publicados entre el 31 de diciembre de 2001 (fecha en la que data la primera noticia en torno a PISA) y el 2 de diciembre de 2013. Para la recogida de datos se utilizó el complemento NCapture para Google Chrome, herramienta del software de análisis cualitativo NVivo10, cuya función era la captura y digitalización de las noticias en formato PDF.

\section{Análisis de los datos}

Una vez recogidos los datos, se procedió a su categorización en el software NVivo10, con el cual se llevó a cabo la clasificación de los datos atendiendo a las siguientes variables:

- Fecha de publicación.

- Trimestre del año.

- Año de publicación.

- Tipología textual.

- Autor.

- Sector del autor.

- Sector del entrevistado.

- Ciclo de PISA.

- Competencia a la cual hace referencia el artículo.

Para analizar la evolución de las noticias, se ha dividido la muestra en cuatro ciclos, coincidiendo con los ciclos completos de PISA a partir del día de la publicación de cada uno de los informes y hasta el día anterior de la publicación del siguiente informe. Así pues, las fechas son:

- $1^{\text {er }}$ ciclo (PISA 2000 - Lectura): del 7 de diciembre de 2001 al 6 de diciembre de 2004.

- $2^{\circ}$ ciclo (PISA 2003 - Matemáticas): del 7 de diciembre de 2004 al 3 de diciembre de 2007.

- $3^{\text {er }}$ ciclo (PISA 2006 - Ciencias): del 4 de diciembre de 2007 al 6 de diciembre de 2010.

- $4^{\circ}$ ciclo (PISA 2009 - Lectura): del 7 de diciembre de 2010 al 2 de diciembre de 2013. 
Una vez realizada dicha categorización, se trasladaron los datos, para su descriptivo, al programa de análisis estadístico SPSS v21.0, utilizando las mismas variables empleadas en NVivo10.

\section{Resultados}

\section{Subgéneros periodísticos y autores}

Siguiendo a Gomis (2008) diferenciamos entre: periodismo informativo-descriptivo, representado por las noticias, los reportajes y las entrevistas; y periodismo interpretativo o de opinión, el cual engloba los artículos de opinión, las editoriales, las columnas, las cartas al director y la opinión del lector.

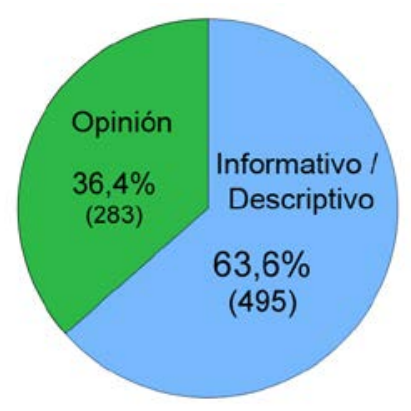

Figura 1. Porcentaje de artículos en función de su intención comunicativa.

Como se observa en la figura 1 , los artículos de carácter informativo-descriptivo tienen una mayor presencia en la muestra analizada, representando casi dos tercios de la misma (63,6\%); mientras que los artículos de opinión suponen el 36,4\% de las mismas. Dichos datos reflejan el predominio de los artículos de corte informativo con respecto a los de opinión, algo que ha venido siendo la tónica general en el periodismo a nivel mundial desde la Segunda Guerra Mundial (de Fontcuberta, 2011).

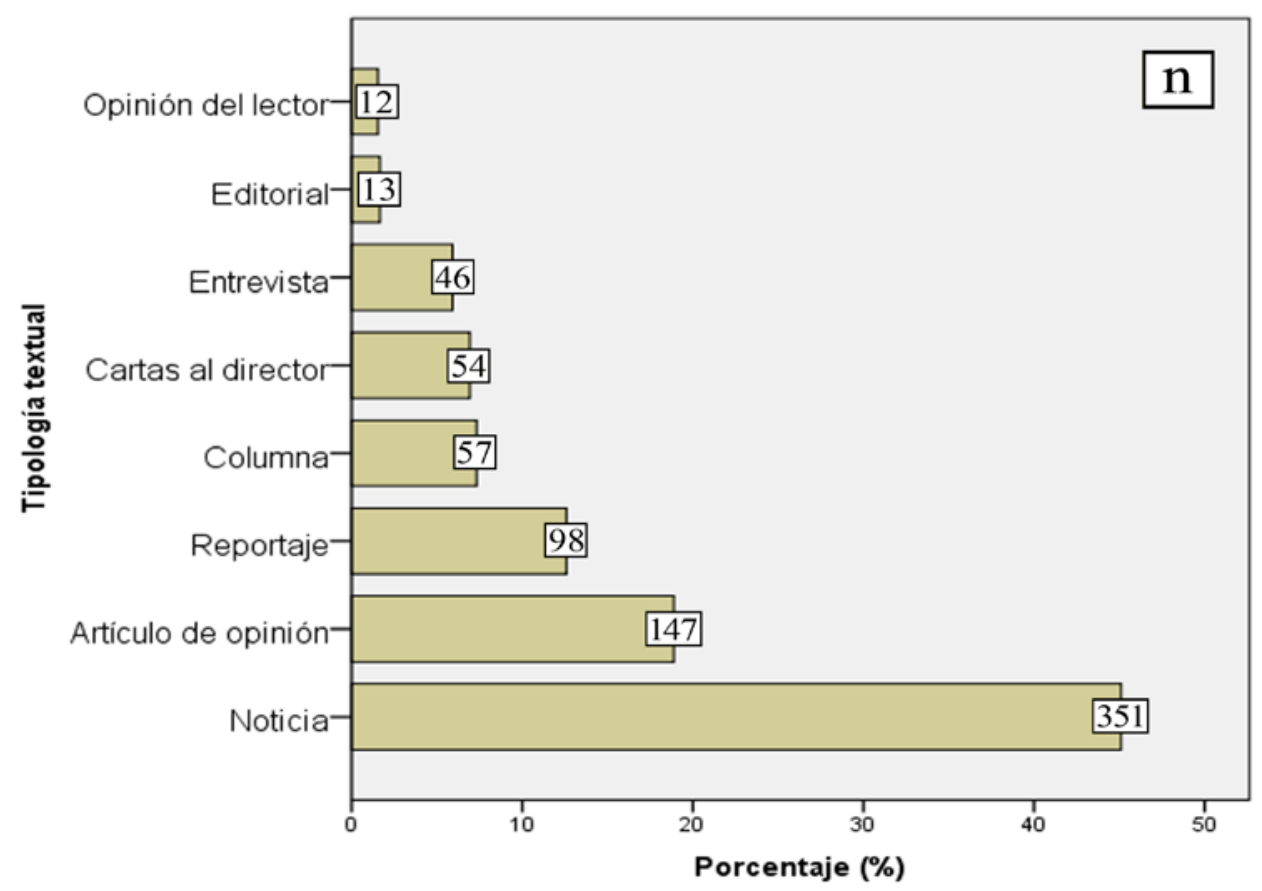

Figura 2. Subgéneros periodísticos utilizados para referirse a PISA. 
Centrándonos en los subgéneros periodísticos, podemos observar en la figura 2 que el género periodístico más numeroso son las noticias, con un 45,1\% (351) del total de artículos, mientras que los artículos de opinión y los reportajes representan un 18,9\% (147) y un $12,6 \%$ (98) respectivamente. Por otro lado, encontramos que las columnas representan el $7,3 \%$ de los artículos con 57 evidencias, seguido de las cartas al director (54) y las entrevistas (46). Las editoriales y las opiniones del lector representan el género periodístico menos frecuente para hablar de la evaluación PISA, con 13 y 12 registros respectivamente.

Estos datos ponen de manifiesto cómo las noticias suponen buena parte del total de los artículos que se refieren a PISA, siendo el género periodístico más utilizado para exponer los aspectos relativos a la evaluación desde un punto de vista descriptivo. Un dato que puede llamar la atención es la poca incidencia de artículos de opinión del lector, que puede deberse al desinterés por parte de los lectores por los asuntos relacionados con PISA o, por el contrario, a que la opinión de la ciudadanía sobre este tema no interesa a los medios.

Respecto al sector de actividad de los autores, el 71\% (551) del total de los artículos fueron publicados por periodistas, diferenciando entre aquellos escritos por periodistas no especializados, con un porcentaje del 58,1\% (452) de los artículos; y aquellos escritos por periodistas especializados en educación, con el 12,7\% (99 artículos), todos ellos escritos por el mismo autor, responsable del área de educación del periódico analizado (J. A. Aunión). Por otro lado, cabe destacar que el 9,8\% (76) de los artículos corresponden a profesores universitarios, mientras que el $7,6 \%$ (59) fueron escritos por diversos autores de diversos ámbitos profesionales como: escritores (39), sociólogos (5), politólogos (3), bibliotecarios (3), abogados (2), lingüistas (2), historiadores (2), investigadores (1), economistas (1) y empresarios (1).

Por otro lado, cabe destacar que todos los artículos de corte informativo fueron escritos por periodistas, quedando los artículos de opinión para los autores provenientes de otros ámbitos y sectores citados anteriormente. No obstante, algunos periodistas escribieron el 19,8\% (56) del total de los artículos de opinión y un 7,2\% del total de los artículos. Por otro lado, debe resaltarse el reducido número de artículos escritos por el profesorado de Primaria y Secundaria (los más próximos a los participantes objeto de estudio), sumando 30 artículos (un 3,9\% del total) entre las dos categorías.

Por el contrario, se puede observar que cuando el periódico acude a fuentes externas, mayoritariamente recurre a personalidades del ámbito político. Así pues, tal y como se puede observar en la figura 3, un 39,1\% (18) de las entrevistas fueron realizadas a personas relacionadas con la política, mientras que las entrevistas a miembros de la OCDE y otras entidades ocupan el segundo y tercer lugar, con un $19,6 \%$ (9) y un $15,2 \%$ (7) respectivamente. Por otro lado, se puede observar que el porcentaje de entrevistados pertenecientes al colectivo de profesorado de Secundaria (un 10,9\% de las entrevistas con 5 registros) es mayor que el de otros colectivos como el profesorado universitario (8,7\%). A pesar de que el profesorado de Secundaria participa menos como firmantes de artículos de opinión (8,9\% de éstos, 26 registros) con respecto al profesorado universitario (25,9\%, 76 registros), se le tiene más en cuenta a la hora de ser entrevistado. Esto podría tener su explicación en el protagonismo principal que tienen los profesores de Educación Secundaria a la hora de explicar los resultados de PISA. 


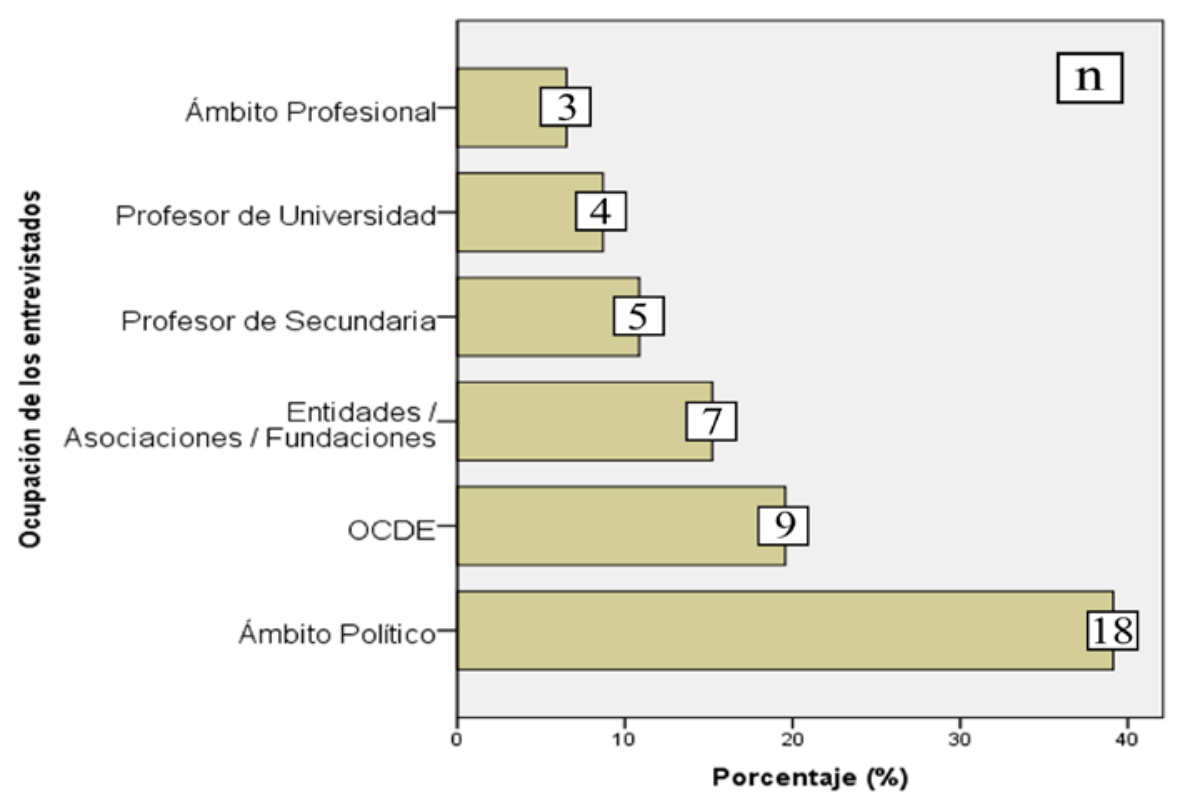

Figura 3. Sector de actividad de los entrevistados.

\section{Evolución de las noticias}

En la figura 4 se puede apreciar la evolución del número de publicaciones entre el año 2001 y 2013 (hasta el 2 de diciembre). Por una parte, se observa que los años en los que más se publica son los años posteriores a la publicación del informe (2002, 2005, 2008, 2011). Debe tenerse en cuenta que el informe se publica en diciembre. Por otra parte, el número de publicaciones en torno a PISA ha aumentado progresivamente desde 2001 a 2008 pasando de 1 a 122 publicaciones respectivamente. Por último, entre los años 2009 y 2013 podemos apreciar un descenso en el número de noticias publicadas, alcanzándose el pico evolutivo de este periodo en el año 2011 con 90 artículos, 32 menos que los registrados en 2008.

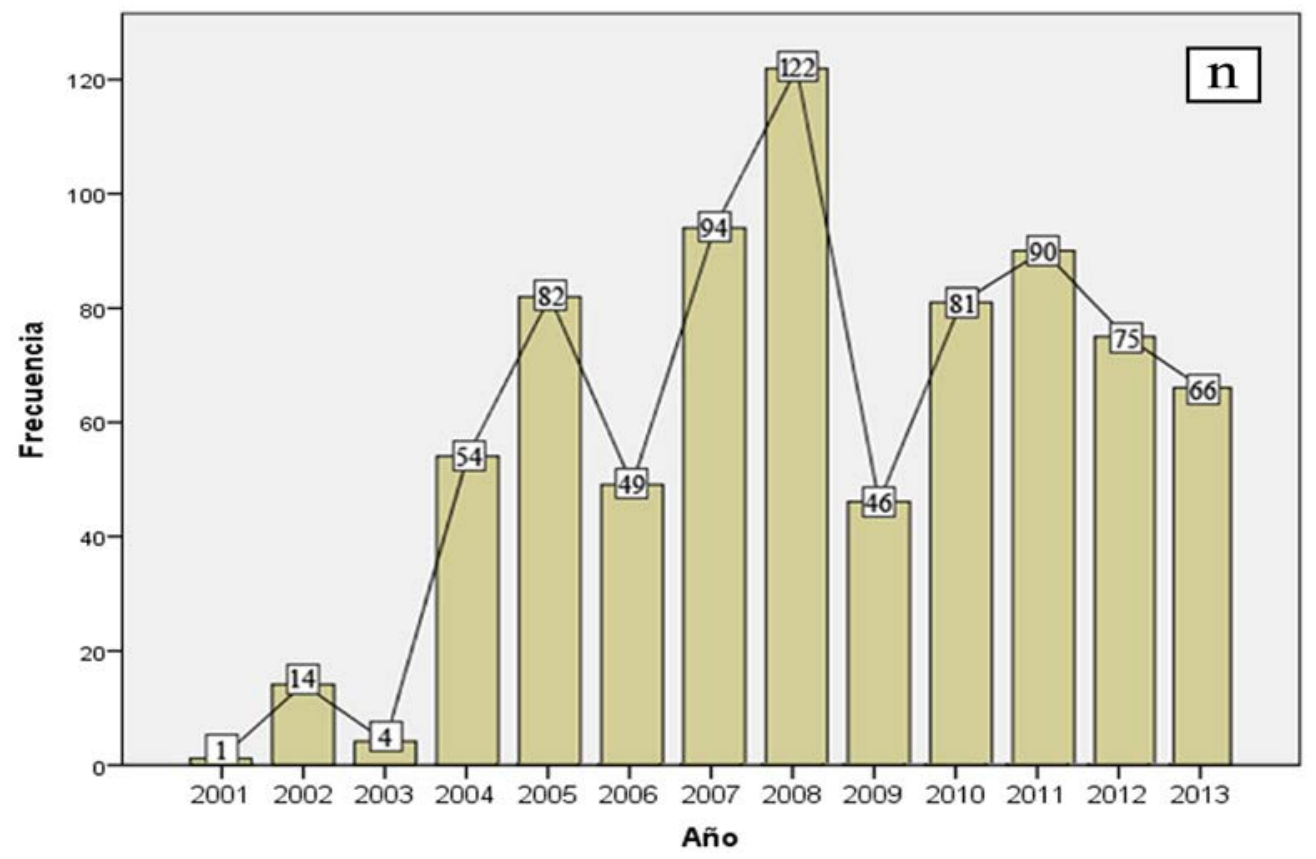

Figura 4. Evolución del número de publicaciones en función del año. 
Para describir la tendencia informativa por ciclos PISA hemos analizado la frecuencia de publicaciones en función de los años naturales, dentro de cada uno de los ciclos de tres años que hay entre la publicación de los resultados de los diversos estudios. Teniendo en cuenta la fecha exacta de publicación de los mismos, la consideración de los años naturales la hemos realizado contando doce meses desde el día exacto del mes de diciembre (por ejemplo: para el Informe 2003 consideramos como 1er año del ciclo desde el día 7 de diciembre de 2004 hasta el 6 de diciembre de 2005, como $2^{\circ}$ año desde el 7 de diciembre de 2005 hasta el 6 de diciembre de 2006, y como 3er año del ciclo desde el 7 de diciembre de 2006 hasta el 3 de diciembre del 2007, ya que al día siguiente se publican los resultados correspondientes a otro ciclo). Dicha frecuencia de artículos por ciclos PISA se puede observar en la figura 5.

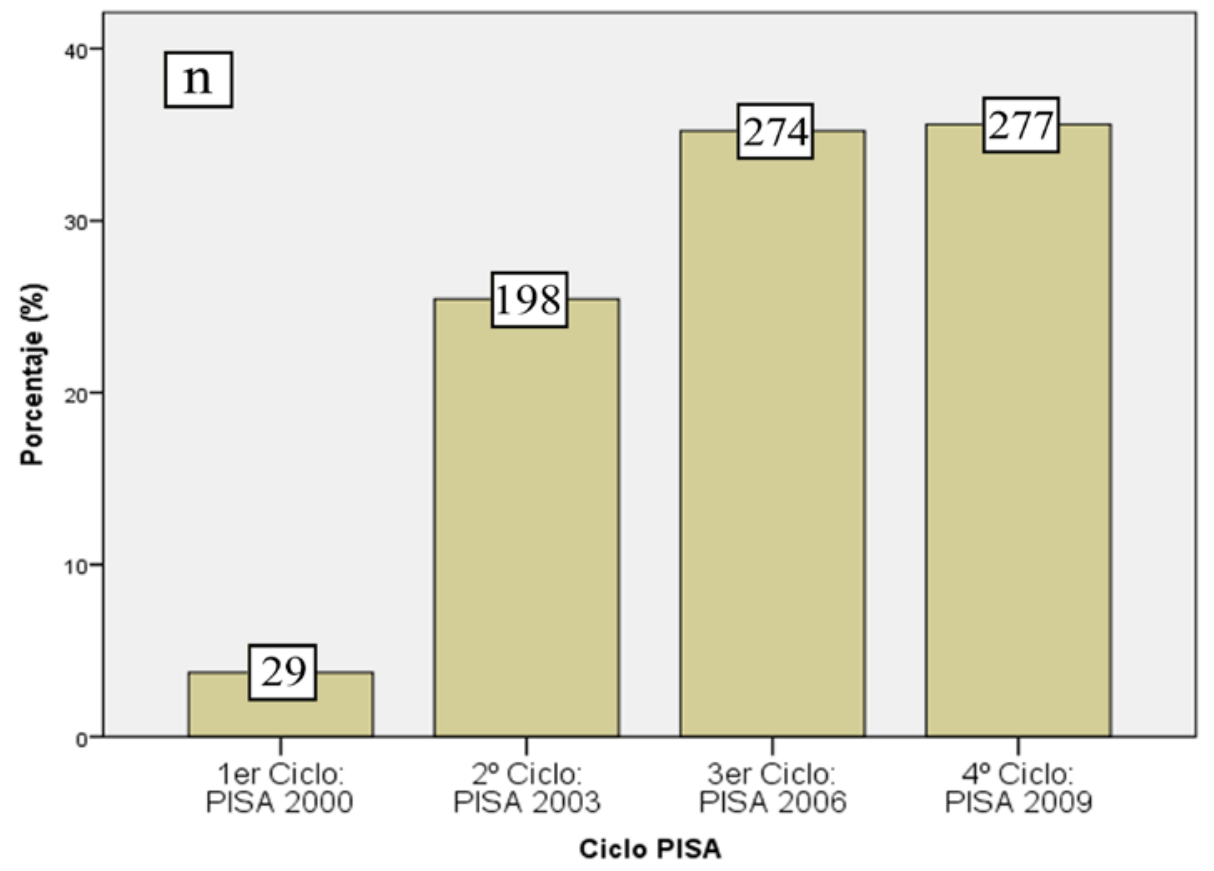

Figura 5. Evolución del número de publicación en función del ciclo PISA.

En el primer ciclo, correspondiente a los resultados de PISA 2000, se observa que tan sólo se publicaron 29 artículos (un 3,7\% del total), aumentando éstos progresivamente en el ciclo de PISA 2003 (198 registros, 25,4\%) y en PISA 2006 (274 registros, 35,2\%), manteniéndose dicho porcentaje en el ciclo de PISA 2009 con cifras muy similares (277 artículos, 35,6\%). Este hecho demuestra que, a juzgar por la frecuencia informativa, el interés por los resultados de PISA por parte de la prensa ha aumentado progresivamente entre los ciclos PISA 2000 y PISA 2006, quedándose estancado en PISA 2009 con cifras similares al ciclo anterior.

En cuanto a la distribución por años dentro de un ciclo, en la figura 6 se puede ver que el primer año a partir de la publicación de los resultados es en el que más publicaciones encontramos, con un 60\% (467) del total de los artículos; mientras que en los dos años siguientes el porcentaje desciende, con un $22,5 \% \quad(175) \quad y \quad$ un $17,5 \%$ 


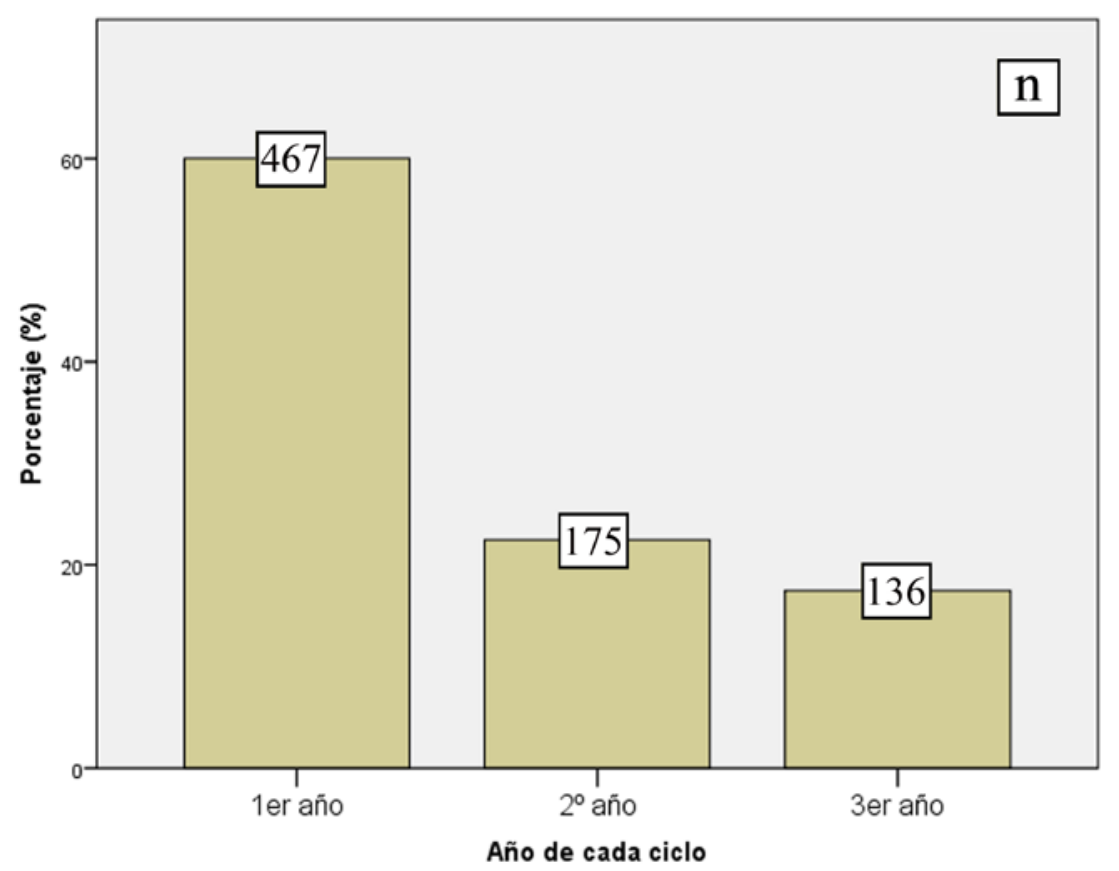

Figura 6. Número de artículos publicados en cada uno de los años del ciclo

Analizando el género periodístico por años dentro del ciclo, en la tabla 1 se puede apreciar que en el primer año se han escrito un total de 285 artículos de corte informativo (61\% del total del primer año), aumentando dicho porcentaje progresivamente en el $2^{\circ}$ y $3^{\text {er }}$ año (con un 66,3\% y un 69,1\% respectivamente). Esto significa que el número de artículos de opinión en el primer año es mayor que en los años sucesivos, con 182 entradas (un 39\%), disminuyendo progresivamente en el $2^{\circ}$ $(33,7 \%)$ y en el $3^{\circ}(30,9 \%)$.

Tabla 1. Relación entre el género periodístico y el año del ciclo

\begin{tabular}{lcccccc}
\hline & \multicolumn{2}{c}{$\mathbf{1}^{\text {er }}$ año } & \multicolumn{2}{c}{$\mathbf{2}^{\circ}$ año } & \multicolumn{2}{c}{ 3 $^{\text {er }}$ año } \\
\hline & $n$ & $\%$ & $n$ & $\%$ & $n$ & $\%$ \\
\hline $\begin{array}{l}\text { Informativo/ Descriptivo } \\
\text { Opinión }\end{array}$ & 285 & 61,0 & 116 & 66,3 & 94 & 69,1 \\
& 182 & 39,0 & 59 & 33,7 & 42 & 30,9 \\
\hline Total & $\mathbf{4 6 7}$ & $\mathbf{1 0 0}$ & $\mathbf{1 7 5}$ & $\mathbf{1 0 0}$ & $\mathbf{1 3 6}$ & $\mathbf{1 0 0}$ \\
\hline
\end{tabular}

Para ilustrar la evolución anual de las publicaciones sobre PISA hemos llevado a cabo un análisis del porcentaje por trimestres, teniendo también en cuenta el primer trimestre de 2014. Como podemos ver en la figura 7, el mayor número de noticias se produce en el cuarto trimestre con 326 registros (43,7\%) y en el primer trimestre con 194 textos (25,4\%); mientras que en los trimestres segundo y tercero el número de noticias desciende, con un $18,6 \%$ y un $12,2 \%$ respectivamente. La explicación gira en torno a la publicación de los resultados de PISA en el mes de diciembre y el posterior rastro informativo reflejado en el primer trimestre del año siguiente. 


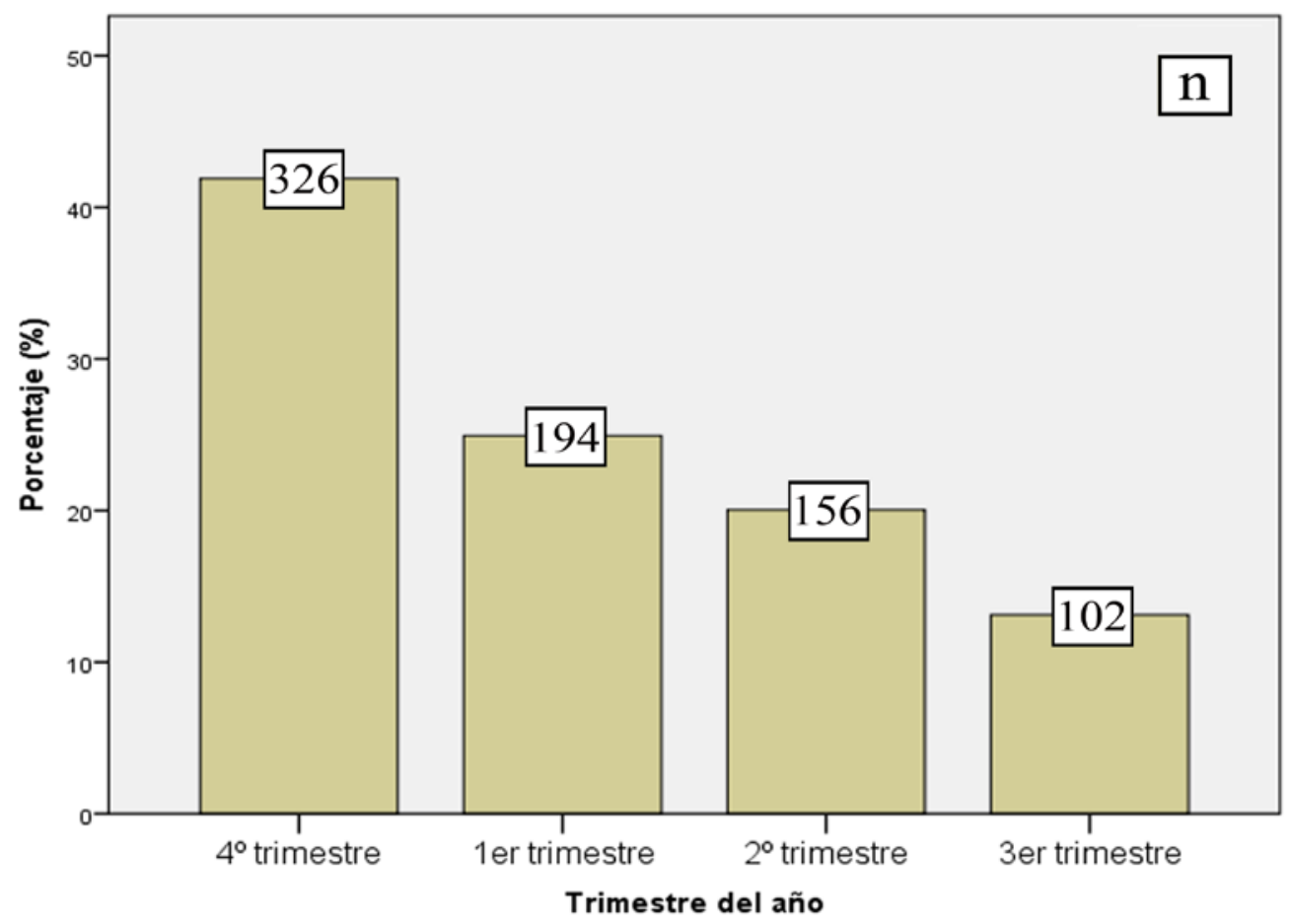

Figura 7. Número de artículos publicados en cada uno de los trimestres del año

Un hecho que debe señalarse en el presente estudio fue el acontecido en los días previos a la publicación de los resultados de PISA 2006, previstos para el 4 de diciembre de 2007, y que desembocó en una sanción para España por parte de la OCDE. Tal y como se refleja en un comunicado emitido por el Ministerio de Educación y Ciencia, el día 28 de noviembre de 2007 se produjo una filtración de los resultados de PISA 2006 en un periódico especializado en educación, rompiendo España el compromiso de confidencialidad firmado con la OCDE ${ }^{2}$. Por ello, se ha llevado a cabo un conteo de los artículos publicados entre el día 28 de noviembre de los años de publicación de los resultados de PISA y el día antes a la fecha exacta de publicación, con el fin de determinar si dicho tema tuvo trascendencia entre la opinión pública.
Como se puede observar en la tabla 2, la prensa se hizo eco de la noticia de las filtraciones de los resultados de PISA 2006, puesto que entre el 28 de noviembre y el 3 de diciembre de 2007 se encontraron 11 artículos (1,4\% del total de la muestra), mientras que en este mismo periodo en el 2004 y el 2010 los porcentajes eran de $0,2 \%$ y $0,9 \%$ respectivamente. Se puede afirmar, por tanto, que las filtraciones llegaron a la opinión pública, tema que generó la inmediata respuesta por parte del Gobierno mediante la publicación del comunicado citado anteriormente. 
Tabla 2. Número de artículos entre el 28 de noviembre y el 3 de diciembre de los años de presentación de resultados

\begin{tabular}{llc}
\hline & n & \% del total \\
\hline Entre el 28 de noviembre y el 3 de diciembre de 2004 & 2 & 0,2 \\
Entre el 28 de noviembre y el 3 de diciembre de 2007 & 11 & 1,4 \\
Entre el 28 de noviembre y el 3 de diciembre de 2010 & 7 & 0,9 \\
\hline Total & $\mathbf{2 0}$ & $\mathbf{2 , 6}$ \\
\hline
\end{tabular}

\section{Competencias evaluadas}

Respecto a las competencias que evalúa PISA, la figura 8 muestra que el 59,3\% (461) de los artículos analizados no citan ninguna de ellas, mientras que en el restante 40,7\% (317) sí se hace referencia a alguna de estas competencias. Esto refleja que las puntuaciones obtenidas en una competencia específica no es el discurso principal a la hora de hablar de los resultados de PISA sino que muchas veces se presentan los artículos de forma descontextualizada y simple, ofreciendo una visión global de los resultados sin fundamentar las opiniones vertidas con datos empíricos y concretos. Estos resultados avalan estudios previos llevados a cabo por Vélaz de Medrano (2006), Pedró (2012) o Domínguez (2015).

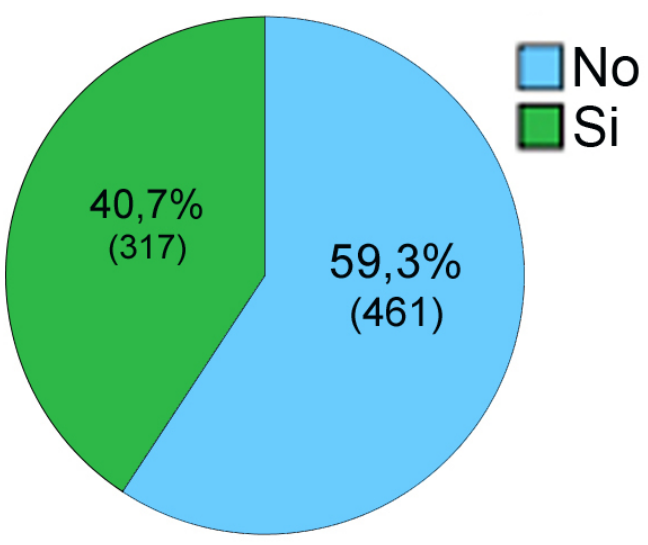

Figura 8. Porcentaje de artículos en función de si hacen referencia a las competencias.

Como se refleja en la tabla 3, de los 317 artículos que hacen mención a alguna de las competencias evaluadas por PISA, un 44,5\% (141) de estos cita a las tres (comprensión lectora, competencia matemática y competencia científica), ofreciendo a los lectores del periódico una visión integral de lo que PISA representa en cuestión de competencias. La lectora es la competencia de la que más se ha hecho eco la prensa, con un total de 67 artículos (21,1\%), seguida de la agrupación lectura y matemáticas con 45 registros $(14,2 \%)$ y de las matemáticas en solitario con 33 artículos (10,4\%). El grupo matemáticas y ciencias lo encontramos en 14 artículos (4,4\%), llamándonos la atención que la competencia científica aparezca más vinculada con las matemáticas que como competencia individual, puesto que tan sólo 9 registros $(2,8 \%)$ hablan de las ciencias de manera exclusiva. Por último, llama la atención que es el agrupamiento entre lectura y ciencias al que menos referencia se hace, con 8 artículos (2,5\%). 
Gracias a estos datos podemos establecer un orden de prioridades mostradas por la prensa al resto de la sociedad en cuanto a la relevancia y la importancia de unas competencias y otras, otorgándole un mayor relieve a la comprensión lectora con respecto a las matemáticas o a las ciencias.

Tabla 3. Número de artículos según la competencia a la que se refiera

\begin{tabular}{lcc}
\hline & n & \% \\
\hline Las tres competencias & 141 & 44,5 \\
Lectura & 67 & 21,1 \\
Lectura y matemáticas & 45 & 14,2 \\
Matemáticas & 33 & 10,4 \\
Matemáticas y ciencias & 14 & 4,4 \\
Ciencias & 9 & 2,8 \\
Lectura y ciencias & 8 & 2,5 \\
\hline Total & $\mathbf{3 1 7}$ & $\mathbf{1 0 0}$ \\
\hline
\end{tabular}

Para incidir en los datos anteriores de una forma más concreta, se ha elaborado la tabla 4, en la cual se muestra el número de artículos teniendo en cuenta las respuestas múltiples, es decir, con independencia de las agrupaciones de competencias que se hayan hecho en los artículos.

Tabla 4. Número de artículos que hablan de cada una de las competencias atendiendo a conjuntos de respuestas múltiples

\begin{tabular}{lc}
\hline & $\mathbf{n}$ \\
\hline Lectura & 261 \\
Matemáticas & 233 \\
Ciencias & 172 \\
\hline
\end{tabular}

Como ilustra la figura 9, los artículos que abordan el tema de las competencias dan un mayor tratamiento informativo a la competencia denominada como principal en cada ciclo de PISA con respecto a las otras en el momento de la publicación de resultados. Así pues, encontramos que en el año 2004 (año de publicación de los resultados al estudio del 2003) son las matemáticas la competencia que más se cita en los artículos (32), mientras que se produce un ligero descenso de registros (28) el siguiente año en favor de la comprensión lectora, que se mantiene con la misma cifra en 2004 y en 2005 (30). 


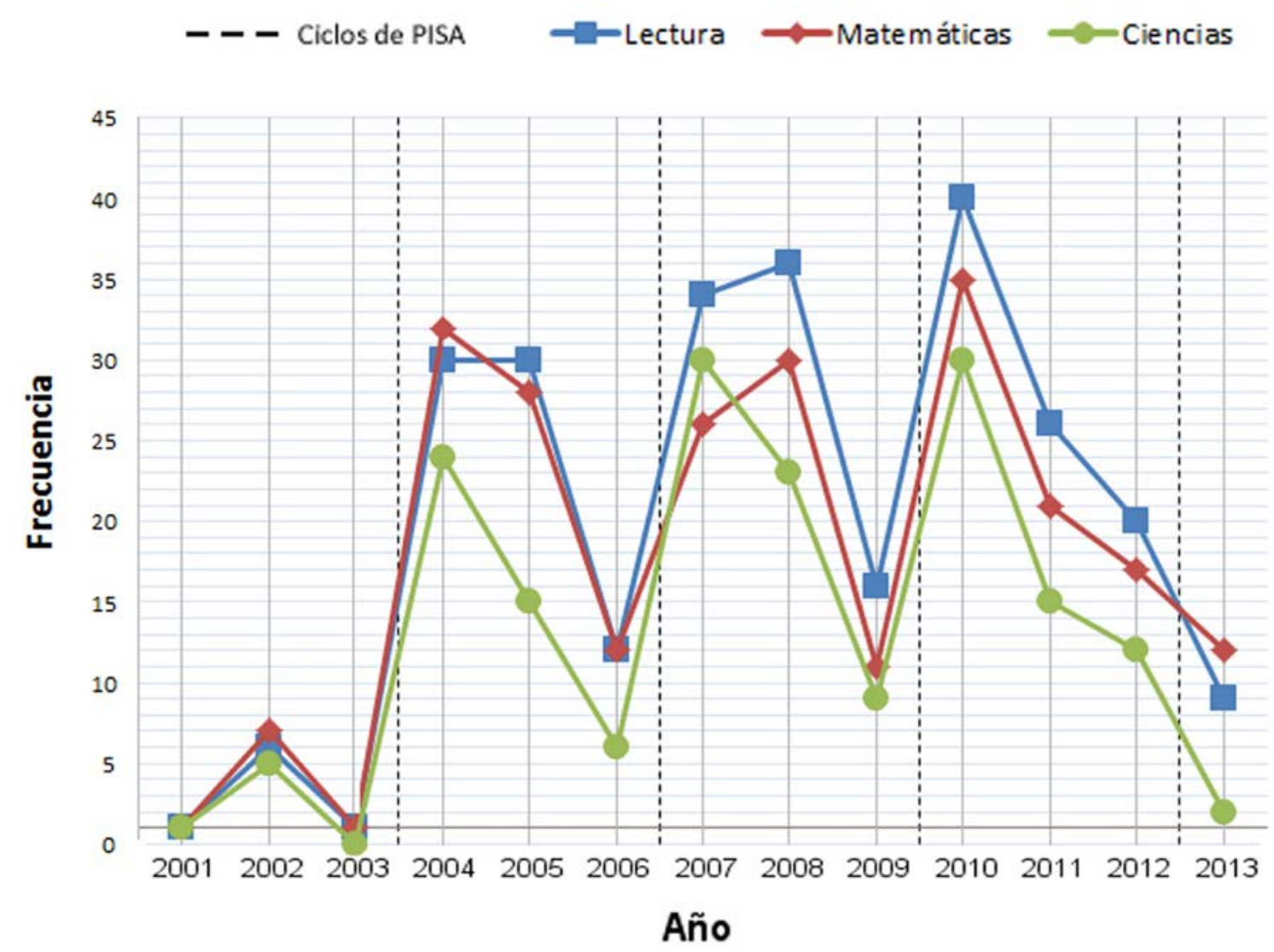

Figura 9. Distribución de las competencias citadas por los artículos en función del año.

Sin embargo, en el ciclo de resultados correspondiente al informe del 2006, y a pesar de que la competencia evaluada como foco principal fueron las ciencias, encontramos que a lo largo del ciclo la comprensión lectora ocupó la mayor parte de las noticias que hacían referencia a alguna competencia. Observamos que en el año 2007 fueron 34 los artículos referidos a lectura, mientras que las competencias matemática y científica se quedaron en 26 y 30 respectivamente. En el año 2008 la cifra de artículos centrados en lectura aumentó hasta 36, al igual que los referidos a la competencia matemática (30); mientras que los dedicados a la competencia científica, siendo la competencia central de dicho informe, descendió hasta los 23 registros. Esta tendencia se repetiría en el año 2009, aunque con muchos menos registros (16 en comprensión lectora, 11 en matemáticas y 9 en ciencias). Este fenómeno de la importancia dada a la competencia lectora requiere un análisis específico.

En la figura 10 se puede apreciar la evolución de los resultados españoles en comparación con los resultados promedio de los países OCDE desde 2000 hasta 2012 en comprensión lectora (Ministerio de Educación, Cultura y Deporte, 2013). En los años intermedios se observa que dicha evolución ha sido diferente a la de otros países, ya que entre 2003 y 2006 el promedio español descendió 20 puntos (de 481 a 461) mientras que el promedio OCDE tan sólo descendió 2 puntos en el mismo periodo (de 494 a 492). Sin embargo, los 20 puntos han sido recuperados entre 2006 y 2009, e incluso incrementados en 7 puntos más entre 2009 y 2012, pasando de 481 a 488. 


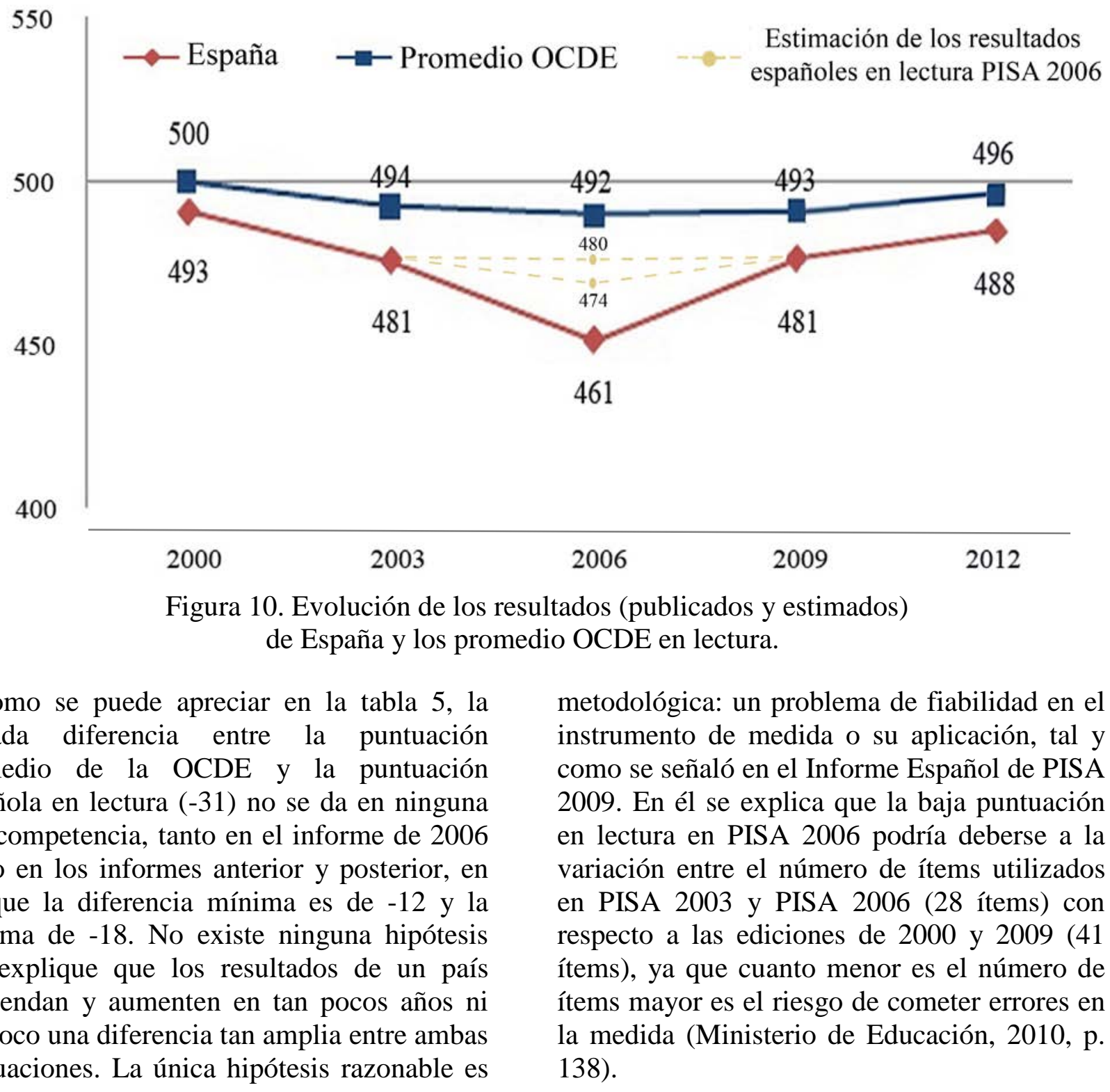

Tabla 5. Puntuaciones promedio OCDE y españolas en lectura, matemáticas y ciencias, y diferencia de puntuación entre ambas

\begin{tabular}{lccc}
\hline & PISA 2003 & PISA 2006 & PISA 2009 \\
\hline Promedio OCDE en lectura & 494 & 492 & 493 \\
Promedio OCDE en matemáticas & 500 & 498 & 496 \\
Promedio OCDE en ciencias & 500 & 500 & 501 \\
\hline Puntuación España en lectura & 481 & 461 & 481 \\
Puntuación España en matemáticas & 485 & 480 & 483 \\
Puntuación España en ciencias & 487 & 488 & 488 \\
\hline Diferencia de puntuación España - OCDE lectura & -13 & $\mathbf{- 3 1}$ & -12 \\
Diferencia de puntuación España - OCDE matemáticas & -15 & -18 & -13 \\
Diferencia de puntuación España - OCDE ciencias & -13 & -12 & -13 \\
\hline
\end{tabular}


Por otro lado, cabe destacar que en el propio Informe Español de PISA 2012 se eliminan los datos de PISA 2003 y PISA 2006 en el apartado correspondiente a la evolución de los resultados, lo que parece señalar el reconocimiento de que no son lo suficientemente fiables (Ministerio de Educación, Cultura y Deporte, 2013, p. 191).

Por último, se ha llevado a cabo una estimación del resultado español en lectura, teniendo en cuenta los máximos y mínimos de las diferencias en las tres competencias evaluadas tanto en PISA 2006 como en PISA 2003 y PISA 2009, y el promedio OCDE en lectura en PISA 2006. Así pues, la puntuación en comprensión lectora debería haber estado entre los 474 y los 480 puntos (figura 10).

Como consecuencia, tenemos, por una parte, que el elevado número de artículos sobre lectura fueron una reacción a los malos resultados en el informe de 2006 y, por otra, que los malos resultados que provocaron dicha reacción no corresponden con la realidad de nivel de comprensión lectora del país.

\section{Discusión y conclusiones}

El impacto de los informes PISA en prensa ha sido importante y creciente desde su primera edición. La mayor parte de las noticias publicadas que hacen referencia a PISA son noticias informativas que describen los resultados de la propia evaluación, mientras que tan sólo un tercio del total de los artículos registrados son de opinión. Los artículos de opinión están firmados por personas de diferentes sectores, como periodistas, personas del ámbito universitario o personas del ámbito profesional. Sin embargo, la opinión de otros sectores como el cuerpo docente de Educación Primaria y Secundaria apenas tienen presencia en la autoría de dichos artículos. No obstante, sí se ha encontrado profesorado de Secundaria entre las personas entrevistadas, consideradas como una de las partes responsables e implicadas en los resultados de las evaluaciones. Respecto a la escasa participación de lectores anónimos, cabría preguntarnos si esta tendencia sigue la línea natural descrita por Navarro (2001) en cuanto a que casi ningún medio de comunicación impreso logra una interactividad entre emisor y receptor o, por el contrario, son los asuntos relacionados con PISA los que no interesan al grueso de la ciudadanía.

Respecto a la evolución de la publicación de noticias, podemos afirmar que el tratamiento de PISA en la prensa $\mathrm{y}$, por lo tanto, la exposición de este tema a la sociedad, ha aumentado considerablemente desde sus inicios y especialmente a partir de los resultados del 2003. Se ha observado que fue con PISA 2006 cuando se publicó un mayor número de artículos, coincidiendo con las bajas puntuaciones en comprensión lectora; mientras que en los años sucesivos, dicho patrón de publicaciones se ha estabilizado. En cuanto al número de artículos a lo largo del año, existe una tendencia estable, ya que mayoritariamente se publica en el mismo trimestre que son presentados los informes de resultados, reduciéndose de forma progresiva a lo largo del año natural. Además, se ha observado una distribución proporcional de artículos de corte informativo y de opinión en cada uno de los momentos del ciclo, no encontrando una mayor incidencia de los de opinión respecto a los informativos en ningún momento.

En cuanto al análisis de las tres principales competencias evaluadas, se ha encontrado que un alto porcentaje de las referencias a PISA hacen un uso general de sus resultados, sin una argumentación o mención específica sobre cada competencia. Este tipo de artículos ofrece una información a la sociedad generalista y poco detallada sobre los mismos, algo alejado de los propios objetivos de estas evaluaciones. Respecto a los artículos que sí hacen referencia a las competencias, hemos encontrado que la comprensión lectora es la que provoca una mayor preocupación, frente a la competencia matemática y la científica, incluso cuando el foco principal de PISA es la evaluación de estas otras competencias.

En este sentido, debe analizarse con detalle lo sucedido a este respecto con el informe del 
2006, en el que los resultados negativos en comprensión lectora provocaron un gran debate. Sin embargo, basándonos en los datos aportados por el informe español de los resultados de PISA 2009 respecto a las limitaciones metodológicas de la evaluación, ponemos en entredicho la fiabilidad del instrumento y la validez de los resultados obtenidos en lectura en la edición de PISA 2006. Unos malos resultados provocaron un amplio debate sobre el nivel de lectura con la comprensible presión a las administraciones educativas para tomar medidas inmediatas para su mejora. Sin embargo, se ha demostrado que los datos que provocaron el debate (y las medidas correctoras) no respondían al nivel real. Este tipo de limitaciones deben hacer reflexionar, además de sobre las puntuaciones reales que deberían haberse obtenido, sobre la necesidad de disponer y utilizar diferentes fuentes de información para el análisis y la toma de decisiones de fenómenos tan complejos como el educativo.

Como reflexión final, podemos concluir que los informes PISA han alimentado de forma clara el debate en torno a la calidad del sistema educativo en España, teniendo la prensa escrita un papel protagonista a la hora de presentar los resultados a la sociedad. Sin embargo, queda demostrado que incluso un estudio con el peso y la trascendencia de PISA puede presentar limitaciones metodológicas, por lo que es necesario que los debates se alimenten de diversas fuentes de información $\mathrm{y}$, en todo caso, se realicen con un uso más prudente de las mismas.

\section{Referencias}

Bautier, E., \& Rayou, P. (2007). What PISA really evaluates: literacy or students' universes of reference? Journal of Educational Change, 8(4), 359-364. doi: http://doi.org/10.1007/s10833-007-9043-9

Calero, J., \& Choi, A. (2012). La evaluación como instrumento de política educativa. Presupuesto y Gasto Público, 67, 29-42.
De Fontcuberta, M. (2011). La noticia. Pistas para percibir el mundo ( $2^{\mathrm{a}}$ ed.). Barcelona: Paidós.

Dominguez, M. J. (2015). El impacto de los proyectos institucionales de evaluación educativa en España (Tesis doctoral). Universidad de León. León.

Dominguez, M., Vieira, M. J., \& Vidal, J. (2012). The impact of the Programme for International Student Assessment on academic journals. Assessment in Education: Principles, Policy \& Practice, 19(4), 393$409 . \quad$ doi: http://doi.org/10.1080/0969594X.2012.659175

Ferrer, F., \& Massot, M. (2005). El proyecto PISA en los medios de comunicación escrita: De la simplificación a la manipulación. Organización y Gestión Educativa, 13(1), 1922.

Gomis, L. (2008). Teoría de los géneros periodísticos. Barcelona: Advisory Board.

Liegmann, A. B., \& van Ackeren, I. (2012). The impact of PIRLS in 12 countries: A comparative summary. En K. Schwippert \& J. Lenkeit (Eds.), Progress in International Reading Literacy in National and International Context (pp. 227-252). Nueva York: Waxmann.

Massot, M., Ferrer, F., \& Ferrer, G. (2006). El estudio PISA y la comunidad educativa: Percepciones y opiniones sobre el proyecto PISA 2000 en España. Revista de Educación, extr(1), 381-398.

Ministerio de Educación (2010). PISA 2009. Informe español. Madrid: Instituto de Evaluación.

Ministerio de Educación, Cultura y Deporte (2013). PISA 2012. Informe español. Volumen I: Resultados y contexto. Madrid: Instituto Nacional de Evaluación Educativa.

Ministerio de Educación, Política Social y Deporte (2008). PISA 2003: Matemáticas. Informe español. Madrid: Instituto de Evaluación.

Ministerio de Educación y Ciencia (2005). Resultados en España del estudio PISA 2000. 
Conocimientos y destrezas de los alumnos de 15 años. Madrid: Instituto Nacional de Evaluación y Calidad del Sistema Educativo.

Ministerio de Educación y Ciencia (2007). PISA 2006: Programa para la Evaluación Internacional de Alumnos de la OCDE. Informe español. Madrid: Instituto de Evaluación.

Navarro, L. (2001). Los periódicos online: sus características, sus periodistas y sus lectores. Sala de Prensa. Recuperado de http://goo.gl/O7ey9h

OCDE (2002). Conocimientos y aptitudes para la vida: Primeros resultados del Programa Internacional de Evaluación de Estudiantes (PISA) 2000 de la OCDE. París: OECD Publishing.

Pedro, F. (2012). Deconstruyendo los puentes de PISA: del análisis de resultados a la prescripción política. Revista Española de Educación Comparada, 19, 139-172. doi: https://doi.org/10.5944/reec.19.2012.7581

Perez, A., \& Soto, E. (2011). Luces y sombras de PISA. Sentido educativo de las evaluaciones externas. Cultura y Educación, 23(2), 171-182. doi: http://doi.org/10.1174/113564011795944758

Pons, X. (2012). Going beyond the 'PISA Shock' discourse: an analysis of the cognitive reception of PISA in six European countries, 2001-2008. European Educational Research Journal, 11(2), 206-226. doi: https://doi.org/10.2304/eerj.2012.11.2.206

Robert, B. (2010). The Europeanization of Education Policies: a research agenda. European Educational Research Journal, 9(4), 519-524. doi: http://doi.org/10.2304/eerj.2010.9.4.519
Rutkowski, L., \& Rutkowski, D. (2010). Getting it 'better': The importance of improving background questionnaires in international large-scale assessment. Journal of Curriculum Studies, 42(3), 411-30. doi: http://doi.org/10.1080/00220272.2010.487546

Schleicher, A. (2006). Fundamentos y cuestiones políticas subyacentes al desarrollo de PISA. Revista de Educación, extr(1), 2143.

Schleicher, A. (2016). Desafíos para PISA. RELIEVE, 22(1). doi: http://doi.org/10.7203/relieve.22.1.8429

Simola, H. (2013). El milagro finlandés de PISA: observaciones históricas y sociológicas. Profesorado. Revista del Currículum y Formación del Profesorado, 17(2), 153-169.

Stack, M. (2007). Representing school success and failure: Media coverage of international tests. Policy Futures in Education, 5(1), 100110. doi: https://doi.org/10.2304/pfie.2007.5.1.100

Velaz de Medrano, C. (2006). Una visión integral de las evaluaciones del PISA con especial atención a la participación en España. Revista de Educación, extr(1), 13-18.

\section{Notas}

[1] Índice de resúmenes generales del EGM. (http://goo.gl/F5IGVt).

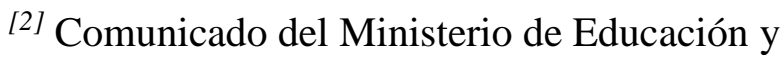
Ciencia sobre la filtración de resultados en el Informe de la OCDE PISA. (http://goo.gl/UGNYc5). 
González-Mayorga, Héctor (hector.glez.mayorga@gmail.com).

Doctorando en el programa de Psicología Educativa y Ciencias de la Educación de la Universidad de León. Licenciado en Psicopedagogía y maestro de Educación Primaria en la especialidad de Pedagogía Terapéutica. Sus líneas de investigación se centran en el estudio del impacto de los estudios PISA en la sociedad española. Dirección postal: Departamento de Psicología, Sociología y Filosofía. Facultad de Educación. Campus de Vegazana s/n. 24071. Universidad de León. León (España).

Vidal, Javier (javier.vidal@unileon.es).

Doctor en Filosofía y profesor titular de la Universidad de León en el área de Métodos de Investigación y Diagnóstico en Educación. Fue Director General de Universidades del Ministerio de Educación y Ciencia español (2006-2008). Sus investigaciones se centran en la educación superior, especialmente evaluación de la investigación, evaluación institucional y relaciones formación-empleo. Ha colaborado con la Comisión Europea. Participa en proyectos internacionales sobre indicadores y tercera misión de la universidad. Dirección postal: Departamento de Psicología, Sociología y Filosofía. Facultad de Educación. Campus de Vegazana s/n. 24071. Universidad de León. León (España).

Vieira, María José (maria.vieira@unileon.es).

Doctora y profesora titular de la Universidad de León en el área de Métodos de Investigación y Diagnóstico en Educación. Sus líneas de investigación se centran en la educación superior en el ámbito internacional, principalmente en la tercera misión de la universidad, su gobierno y la relación universidad-empleo. Ha colaborado con la ANECA, como asesora de la Dirección General de Universidades del MEC (20062009) y con la Comisión Europea en la Estrategia de Modernización de la Educación Superior. Dirección postal: Departamento de Psicología, Sociología y Filosofía. Facultad de Educación. Campus de Vegazana s/n.

ORCID $\underline{0000-0003-1161-1694}$

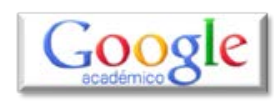

ResearchGate 24071. Universidad de León. León (España).

\section{RELIEVE}

\section{Revista ELectrónica de Investigación y $\mathbf{E} \mathbf{V}$ aluación Educativa E-Journal of Educational Research, Assessment and Evaluation}

[ISSN: 1134-4032]

(C) Copyright, RELIEVE. Reproduction and distribution of this articles it is authorized if the content is no modified and their origin is indicated (RELIEVE Journal, volume, number and electronic address of the document).

(C) Copyright, RELIEVE. Se autoriza la reproducción y distribución de este artículo siempre que no se modifique el contenido y se indique su origen (RELIEVE, volumen, número y dirección electrónica del documento). 